\title{
L'art du faible : de l'utilisation des lieux frontières polémologiques dans le cinéma gay français
}

\author{
Florian Grandena \\ Université d'Ottawa
}

1983. Henri (Jean-Hugues Anglade), un jeune homme, marche un peu gauchement dans les dédales d'une gare anonyme et peu accueillante. Henri parcourt les quais, les couloirs déserts et s'arrête dans des salles d'attente presque vides. Sa flânerie nocturne le mène parfois à la collision avec d'autres corps masculins, parfois inquiets, parfois haletants, croisés au détour d'un coin de couloir ou rencontrés dans les tasses. Et c'est bien dans ces lieux de passage anonymes baignant parfois dans la lumière crue de néons et a priori dénués de tout érotisme 
qu'Henri rencontre l'objet de tous ses désirs, Jean (Vittorio Mezzogiorno), une petite frappe virile, séduisante, manipulatrice et proxénète à ses heures. Une relation faite d'hésitations, de titillements cruels de la part de Jean, d'espérances trahies et de coups bas qui, de maints baisers avortés à des gestes érotiques ou violents, deviendra vite tout aussi obsessionnelle que dangereuse.

1993. La ville nocturne, cette fois clairement identifiée (Paris). Un autre Jean (Cyril Collard), beau jeune Parisien, parcourt avec un ludisme assumé les quais nocturnes de la Seine. Là, il rencontre d'autres hommes, noyés dans l'obscurité, réduits à des silhouettes sombres, rassemblées par petits groupes autour de corps d'hommes disponibles ayant pour objectif une gratification sexuelle facile et immédiate. Échanges de baisers et de gestes, requêtes sexuelles exprimées simplement et directement en quelques mots crus: les quais de la Seine deviennent la scène de la circulation du désir homosexuel décomplexé et de sa concrétisation charnelle par le biais d'un rapport charnel en dehors de toute justification, de tout cadre normatif et moralisateur. Le désir semble libéré, voire libre, mais le jeune et beau Jean nous le rappelle sans cesse : cette liberté apparente dans les lieux nocturnes s'accompagne parfois de ses contraires, à savoir le néo-fascisme par le biais de la présence de skin heads mais aussi le sida, qui n'est jamais très loin (Jean est infecté, il le sait, mais refuse de se laisser affecter).

2004. Nouveau rendez-vous à Paris (Paris, encore et toujours le lieu, dirait-on, de toutes les histoires et de tous les possibles...). Gare du Nord. Un jeune homme, Djamel (Yasmine Belmadi), fend lentement la foule de voyageurs et accoste parfois certains usagers pressés pour demander une cigarette 
et, parfois, avec un peu de chance, un peu plus, à savoir une passe rapide dans les toilettes de la gare. Clients, clientes, Djamel n'est pas regardant, car il est là pour gagner sa vie et non pas pour sa propre gratification sexuelle. Quant à Stéphanie (Stéphanie Michelini) - péripatéticienne transexuelle, qui compose avec Djamel et Mikhail (Edouard Nikitine), jeune russe viril ne connaissant pas un mot de français, un heureux et tendre ménage à trois bisexuel - , elle erre, avec ses amies et collègues de trottoir brésiliennes, dans les espaces nocturnes du bois de Boulogne et tente de négocier une passe avec un client protégé dans le réceptacle de son véhicule chauffé.

Trois films, respectivement L'Homme blessé (Patrice Chéreau, 1983), Les Nuits fauves (Cyril Collard, 1993) et Wild Side (Sébastien Lifshitz, 2004), marquant chacun à leur façon une décennie différente et apportant leur pierre à l'édifice de la culture gay et de ses diverses (auto)représentations. Dans ces œuvres, le personnage gay est enraciné dans des lieux de passage nocturnes empreints, semblerait-il donc, d'une certaine tradition genétienne exigeant de la figure masculine homosexuelle un penchant marqué pour la délinquance, voire pour la criminalité (Provencher, 2002). Ces endroits, nous les appellerons ici des lieux frontières, c'est-à-dire des zones tampons entre la sphère publique, plus précisément le monde diurne de l'hétérosexualité et du capitalisme, et la sphère privée dans laquelle l'homosexualité est souvent reléguée par l'hétérosexualité. Nous nous interrogerons sur la représentation de ces lieux dans le gay noir ainsi que sur leurs fonctions et leur rôle dans, à la fois, l'établissement d'une relation polémologique avec le groupe dominant qu'est l'hétérosexualité et de la réalisation des potentialités de résistance. 


\section{Le lieu frontière comme espace polémologique}

Les trois œuvres cinématographiques considérées ici utilisent d'une façon qui leur est propre de tels lieux et participent ainsi d'une sous-tendance du cinéma à problématique homosexuelle français, à savoir le gay noir (Grandena, 2014). Empruntant, selon les œuvres, à des mouvements cinématographiques connus, tels que le réalisme poétique français et le film noir américain, le gay noir affiche une prédilection pour les décors nocturnes et les bas-fonds urbains qui, malgré leur dangerosité, deviennent l'arrière-scène de sujets masculins désirants. Ici, les pavés sont luisants, les zones d'ombre sont dominantes et la lumière, lorsque lumière il $\mathrm{y}$ a, émanant de lampadaires et autres plafonniers, est souvent blafarde, plongeant tout le décor et les personnages dans un chiaroscuro parfois dramatique... Entretenant une relation ambiguë avec les notions de bien et de mal à l'instar des anti-héros du film noir américain, les personnages gay de ce corpus d'œuvres cinématographiques semblent ne pouvoir (ou peut-être ne vouloir) s'extraire de forces sociales dominantes: ces personnages seraient-ils prédestinés, et incapables d'échapper, à ce qu'ils sont / font? Et si les corps désirants ne cessent de se rapprocher, ils ne se retrouvent pas souvent, voire jamais, l'amour et le sexe, monnayés ou non, ne semblant jamais favoriser un rapprochement autre que furtif et uniquement charnel. Juste le temps d'une étreinte, d'un échange sexuel, de quelques mots échangés, une intimité sans grande intimité en somme ${ }^{1} .$. À

1 À l'opposé du gay noir, existe une autre tendance, celle du gay propre à l'idéologie homonormative et assimilationniste. Les œuvres inclues dans le gay propre (tendance transnationale qui concerne aussi les productions télévisuelles) mettent en avant un désir de normalisation marqué (comme dans le contexte nord-américan Modern Family et The New Normal), tentant 
première vue, le bonheur n'est pas de mise, principalement pour les personnages de L'Homme blessé et des Nuits fauves, où amour et désirs semblent accompagner une psyché sombre et torturée. Henri finira par tuer l'objet de ses désirs² tandis que le protagoniste des Nuits fauves hésitera fort longtemps avant de commencer à s'aimer et de cesser de mettre sa vie en danger comme celle de certains de ses proches. Le lieu frontière est souvent sombre, sale et parfois lugubre, habité et traversé de sentiments violents et contradictoires.

Comment comprendre et définir ici le lieu frontière? C'est, entre autres, un non-lieu. Au sein du gay noir, le désir et le plaisir homosexuels (ainsi que sa recherche ou sa marchandisation, ou les deux à la fois) sont indissociables de ces lieux a priori fondamentalement hétérosexuels ou, plus précisément, fondamentalement hétéronormés et hétéronormatifs, ces endroits précis, nettement définis et prédéterminés par une topographie et une fonction précises. De tels lieux sont, si nous devons suivre la réflexion de Marc Augé, des non-lieux, à savoir aussi bien les installations nécessaires à la circulation accélérée des personnes et des biens (voies rapides, échangeurs, aéroports) que les moyens de transport eux-mêmes ou les grands centres commerciaux, ou encore les camps de transit prolongés où sont parqués les réfugiés de la planète (Augé, 1992, p. 48). Ces non-lieux, avant qu'ils ne soient

de gommer ainsi toute caractéristique subversive liée à l'homosexualité. La dichotomie entre gay noir et gay propre fait volontairement écho à la distinction entre bons et mauvais citoyens sexuels telle que développée par Denis Provencher (2007).

2 D'ailleurs, L'Homme blessé a été conçu par son scénariste, Hervé Guibert, comme un hommage on ne peut plus direct au Journal du voleur de Jean Genet. Les Nuits fauves, d'un autre côté, fait au début de la diégèse une référence directe à la mort de Genet. 
occupés par les protagonistes homosexuels du gay noir, sont traversés et utilisés par maints individus en partance vers leur lieu de travail ou de retour vers leur domicile, marquant ainsi le lieu en question de leurs habitudes, de leurs pratiques et aussi de leur histoire (personnelle, familiale, professionnelle, collective). Ces non-lieux sont donc des endroits pratiqués par leurs usagers (de Certeau, 1980, p. 197-208).

Mais le lieu frontière n'est pas seulement un non-lieu, c'est aussi, d'une certaine façon, un espace polémologique où peuvent s'exprimer les tensions, voire les conflits, entre deux communautés: la communauté hétérosexuelle qui évolue au sein de la sphère publique sans y penser à deux fois (un espace qui devient la scène de l'hétéronormativité par excellence) et la communauté $\mathrm{LGBTTQ}^{3}$ qui, lorsqu'elle désire sortir de la sphère privée à laquelle elle est souvent reléguée, trouve comme lieu d'existence privilégié les lieux urbains nocturnes. Le lieu frontière polémologique, c'est donc un peu le lieu mais aussi l'histoire de groupes qui sont définis selon les pratiques et les rôles sociaux en lien, en partie, avec leur orientation sexuelle et, surtout, avec leur rapport (adhésion et rejet partiels ou totaux) à l'hétéronormativité et qui cherchent et se disputent parfois une place sur un même territoire public (qui appartient à tous et à personne à la fois).

Il existe maints exemples du gay noir dans le paysage cinématographique français : Notre paradis (Gaël Morel, 2011), mettant en scène deux amants criminels, prostitués tous deux, tout comme les protagonistes de Eastern Boys (Robin Campillo, 2013), J'embrasse pas (André Téchiné, 1991), Miss Mona (Mehdi Charef, 1987), Tenue de soirée (Bertrand Blier, 1986) et Tiresia

${ }^{3}$ Lesbienne, gay, bisexuelle, transgenre, transsexuel, queer. 
(Bertrand Bonello, 2002) pour ne citer que ceux-là. Dans le gay noir, nous le savons, l'homosexuel se voit attribuer des lieux frontières de prédilection, détournés de leur fonction première (la circulation des individus et des biens) et privés de leurs usagers habituels (les voyageurs et les travailleurs en transit, les consommateurs, les familles) : les boyaux de gare anonymes, les routes sombres du bois de Boulogne, les quais de la Seine, les trottoirs sombres, les fêtes foraines - en somme, des lieux de passage ou de transit appartenant d'habitude au monde de l'hétérosexualité. Car c'est bien cette dernière qui, habituellement, domine ces lieux impersonnels et permet à l'homme et à la femme hétérosexuels d'afficher ensemble leur complicité amoureuse, leur connivence, leur mariage, leurs enfants, sans problème ou sans arrière-pensée, et ce, à la barbe de ce groupe social qu'est la communauté LGBTTQ.

C'est une remise en question des stratégies hétéronormatives et de la place de celles-ci dans les rapports de force et de pouvoir avec des groupes dominés que tentent d'opérer les braconniers homosexuels dans les films du gay noir. Michel de Certeau nous dit :

J'appelle stratégie le calcul (ou la manipulation) des rapports de force qui devient possible à partir du moment où un sujet de vouloir et de pouvoir (une entreprise, une armée, une cité, une institution politique) est isolable. Elle postule un lieu susceptible d'être circonscrit comme un propre et d'être la base d'où gérer les relations avec une extériorité de cibles ou de menaces (les clients ou les concurrents, les ennemis, la campagne autour de la ville, les objectifs et objets de recherche). (de Certeau, 1980, p. 85)

Une résistance au système dominant et aux utilisations tout aussi hégémoniques qu'hétéronormées qu'il fait de certains endroits, en un temps donné, est possible grâce à des pratiques 
alternatives de ces espaces. Selon les œuvres du gay noir, il s'opérerait donc un phénomène de ré-appropriation et de détournement des lieux, par le biais d'actes sexuels entre personnes de même sexe (ludiques dans certains films, indispensables à la survie financière de la personne dans d'autres), du lieu généralement réservé aux pratiques hétéronormatives. Cet autre espace, de nature polémologique, devient ainsi un lieu frontière existant entre, d'une part, le monde diurne héréronormatif, qui est aussi celui du capitalisme, et, d'autre part, la sphère privée, dans laquelle l'hétéronormativité voudrait cantonner l'homosexualité, permettant de contrôler les apparitions possibles de cette dernière (sans oublier ses expressions politiques et identitaires potentielles au sein de la sphère publique). L'hétéronormativité rattachée à ces espaces est donc mise à mal tandis que le désir gay et sa concrétisation charnelle investissent des lieux publics temporairement libérés d'un joug idéologique visant entre autres au contrôle de l'homosexualité par le biais de son invisibilité forcée et de la surveillance de ses pratiques : dans le gay noir, les gays se frôlent, se touchent et baisent librement. Échanges corporels mais aussi financiers, constituant une économie du désir et de flux financiers qui n'est plus au service de la sphère capitaliste mais qui se situe parallèlement à celle-ci.

À l'instar de de Certeau, on pourrait donc voir, dans ces pratiques spatiales alternatives, une poïtique menant à l'expression d'une résistance culturelle, politique et idéologique par le biais d'attitudes, de gestes et d'actes dont le but serait l'émancipation de certains sujets ou de certains usagers du lieu pratiqué. Ces individus, de par leurs actions de détournement du contrôle et des stratégies de l'espace diurne hétérosexuel, deviennent des braconniers (pour reprendre la terminologie de 
de Certeau) sur un territoire liminal en voie de réappropriation dont les tactiques s'opposeraient aux stratégies du monde hétéronormatif. Pour de Certeau, la tactique « est ruse [...] l'art du faible » (1980, p. 87) et, plus précisément,

l'action calculée que détermine l'absence d'un propre [...]. La tactique n'a pour lieu que celui de l'autre. Aussi doit-elle jouer avec le terrain qui lui est imposé tel que l'organise la loi d'une force étrangère [...]. Elle fait du coup par coup. Elle profite des "occasions" et en dépend, sans base où stocker des bénéfices, augmenter un propre et prévoir des sorties. Ce qu'elle gagne ne se garde pas. (de Certeau, 1980, p. 86-87)

L'espace public diurne est non seulement le lieu de l'hétéronormativité et de ses stratégies de groupe dominant mais aussi, fréquemment, l'endroit de prédilection de la sphère capitaliste : de sa production officielle et reconnue (comme la présence de commerces et l'acte de consommation), de sa circulation (financière mais aussi communicationnelle par le biais de l'omniprésence des nouvelles technologies striant et sillonnant désormais l'espace physique) et de la présence de sa main-d'œuvre (les voyageurs transitant entre leur domicile et leur lieu de travail et vice versa). Car, comme Guy Hocquenghem l'a indiqué, l’on ne peut négliger le rôle joué par la famille dans l'établissement et le maintien de la sphère capitaliste : afin d'assurer sa survie, le capitalisme se doit de contrôler les interconnexions a priori illimitées entre individus en utilisant le complexe d'CEdipe comme principe organisant du désir. Ainsi, ce dernier est canalisé («œdipianisé») de façon stratégique vers une institution essentielle au capitalisme, à savoir la famille (Hocquenghem, 2000, p.91-121) censée reproduire aussi bien la main-d'œuvre que les valeurs essentielles au maintien de l'ordre économique. Un espace hétérosexuel et capitaliste, donc. Mais là, dans ce lieu braconné, 
les tactiques des usagers n'ont pas pour objectif de soutenir ou de cautionner le capitalisme mais de le saper temporairement, c'est-à-dire de le déstabiliser : l'amour entre hommes est une activité qui ne sert pas le système économique; c'est du temps passé à autre chose qu'une activité soutenant le capitalisme (pas de reproduction de la main-d'œuvre, ni de valeurs utilitaristes). C'est, à l'instar de la pratique de la "perruque » décrite par de Certeau4, une perte de temps importante, car contre-productive, une résistance délinquante au système, ellemême création salvatrice d'un espace d'opposition au sein d'une sphère publique dominante.

Comme nous l'avons mentionné en ouverture de ce texte, L'Homme blessé, Les Nuits fauves et Wild Side comportent divers exemples de déstabilisation des rapports de force et de tentatives de résistance de la part d'un groupe dominé (numériquement et idéologiquement) face à un groupe dominant. L'Homme blessé, premier film gay français à proprement parler, mettant en avant, et sans concession aucune, un désir homosexuel revendiqué (mais malheureux), est une histoire sombre dont l'action se déploie dans divers endroits urbains presque interchangeables: les rues, les dessous de pont, une fête foraine et, surtout, les multiples coins et recoins de la gare ferroviaire d'une grande ville non identifiée. Là, Henri, jeune homme fluet à la démarche

\footnotetext{
4 «Accusé de voler, de récupérer du matériel à son profit et d'utiliser les machines pour son compte, le travailleur qui "fait la perruque" soustrait à l'usine du temps (plutôt que des biens, car il n'utilise que des restes) en vue d'un travail libre, créatif et précisément sans profit... Bien loin d'être une régression vers des unités artisanales ou individuelles de production, la perruque réintroduit dans l'espace industriel (c'est-à-dire dans l'ordre présent) les tactiques "populaires" de jadis ou d'ailleurs. » (de Certeau, 1980, p. 70)
} 
maladroite, se prend de passion pour une petite frappe virile et émoustillante, qui ne cessera, pour le malheur du jeune homme et finalement le sien (puisqu'il sera tué par le jeune homme), de trahir Henri et de le berner quant à la véritable nature de leur relation (qui, malgré maints effleurements et caresses, restera chaste). À la suite de sa rencontre violente avec Jean dans les toilettes de la gare (scène durant laquelle Jean frappe un homme et force Henri à faire de même pour ensuite le plaquer contre la porte des WC, corps contre corps, visage contre visage, et l'embrasser violemment, entretenant une ambiguïté fondamentale entre la performance de la violence et celle du désir amoureux), Henri, nouveau membre de la faune nocturne gay, hante les couloirs de gare afin de se rapprocher de Jean et de concrétiser ce désir qui le tenaille. À la mi-chemin du film, nous voyons Henri, taciturne, le regard perdu dans le vide, dévorant symboliquement un sandwich. Puis, sans transition aucune, nous retrouvons le jeune homme assis sur un banc de la salle d'attente, des immondices jonchant le sol, le maillot blanc taché à moitié remonté sur son torse maigre, se lever brusquement pour tenter d'acheter un billet de train. Manquant d'argent et dépité, Henri se dirige alors vers les toilettes. Là, debout devant la pissotière, il regarde les visages de ses voisins de droite et de gauche, tandis que l'un d'eux plonge le regard vers son sexe. Derrière les trois hommes en passent d'autres, les regards des uns caressant les corps des autres (les fesses, le visage). Mais Henri ne semble pas très à l'aise dans les toilettes et en sort rapidement. Après que leurs regards se sont accrochés, Henri propose brusquement à un jeune homme en imperméable (Hammou Graïa) : «si tu me donnes 100 francs, je couche avec toi ». Face au refus de l'homme en imperméable, Henri réplique: "J'ai que 50 francs sur moi, alors je te les 
donne, juste pour un baiser ». S'ensuivent plusieurs plans où les deux jeunes hommes tentent de trouver un endroit tranquille pour un instant d'intimité : trop de gens (uniquement des hommes) dans les couloirs de gare, des sans-abris au détour d'un recoin qui, pourtant, avait l'air si tranquille. Et quand les lèvres des jeunes gens se rencontrent enfin, une première fois, une brève seconde, un cheminot leur crie l'ordre de disparaître. Enfin, dans un couloir sale, Henri et le jeune homme s'embrassent passionnément, puis se séparent après avoir échangé quelques banalités.

Cette séquence de L'Homme blessé est révélatrice de la résistance potentielle (et, dans ce cas, en partie problématique puisque le désir homosexuel ne pourra se concrétiser dans le film) du groupe dominé dans un endroit habituellement traversé et utilisé par des milliers d'individus qui, s'ils le désirent, pourront afficher leur amour, leur relation, si ceux-ci sont hétérosexuels. Ici, la brèche ouverte à la résistance, l'occasion de résister et de braconner sur les terrains du groupe dominant, prend la forme de l'occupation du lieu frontière la nuit, c'est-à-dire au moment où les transits de voyageurs et les activités diverses et variées du capitalisme ralentissent au point de devenir presque invisibles et inaudibles. Pour un seul baiser, bref et monnayé, Henri et le jeune homme doivent ruser, trouver avec difficulté une bulle de tranquillité pouvant éclater à tout moment et n'étant que momentanée, car «les tactiques misent sur une habile utilisation du temps » (de Certeau, 1990, p.63). Vécue et composée dans l'instant, la représentation de cette micro-résistance ne peut être surestimée dans le contexte du film dans son ensemble, L'Homme blessé ne laissant que fort peu d'espace à une résistance autre que courte et directement assujettie à la présence des membres du groupe dominant (des 
hommes hétérosexuels tels que le cheminot). Mais la possibilité de la résistance potentielle, comme sa créativité, est là; elle hante chaque image tout en rendant hommage à cette communauté de destin et de fait ${ }^{5}$ qui a su, à travers les âges, survivre face à une adversité parfois répressive.

\section{Un lieu frontière physique et organique}

L'Homme blessé, Les Nuits fauves et Wild Side suggèrent que ce lieu frontière est à la base constitué par le regard des hommes impliqués. Le regard est le premier contact (parfois presque le seul, comme dans le film de Chéreau) entre les hommes dans les tasses de la gare nocturne; c'est par lui aussi que les individus cherchent à établir une reconnaissance de l'homoïté (c'est-àdire d'une «relation fondée sur le même plutôt que sur une hiérarchie de différences antagonistes », Bersani, 1995, p. 18) et, de par là même, un premier pas dans l'établissement d'une relation interindividuelle (que celle-ci mène à une relation charnelle ou non).

L'Homme blessé, encore: au commencement du film de Chéreau, Henri et sa famille (composée de son père, presque muet et distant; de sa mère, bavarde et stressée; et de sa sœur, bougonne) doivent attendre un train dans une salle d'attente comble. Là, le regard d'Henri et celui, fixe et insistant, d'un homme mystérieux, Bosman (Roland Bertin), un docteur qui servira d'intermédiaire entre Jean et Henri, se croisent.

\footnotetext{
5 Frédéric Martel définit la communauté de destin comme « une communauté virtuelle, quelque chose comme une conscience commune à nombre d'homosexuels et d'abord une mémoire commune» tandis que la communauté de fait se rapporte à «la drague et [à] un folklore autour du désir amoureux » (1996, p. 709-710).
} 
Lorsqu'Henri, timoré, sort précipitamment de la salle d'attente, Bosman le suit jusqu'aux consignes dans le sous-sol de la gare, mais s'enfuit après avoir aperçu Jean, qui offre spontanément une boisson à Henri (en une évocation sensuelle, tous deux partagent la même bouteille). Même si le jeu de regards entre Henri et l'homme plus âgé ne mène pour le moment à rien, il permet la rencontre entre Henri et Jean. En somme, le regard (homo)érotique et (homo)érotisant ainsi que la reconnaissance de l'autre en ce qu'il nous ressemble sont la condition sine qua non de l'établissement du lieu frontière dans le gay noir. Les homosexuels, ces virtuoses du regard 6 ...

Wild Side regorge aussi de scènes où le regard est l'une des expressions des tactiques braconnières et constitutif d'un espace accueillant les pratiques sexuelles du groupe dominé. Tel est le cas des séquences nocturnes qui nous présentent Stéphanie en train de tapiner avec ses amies brésiliennes: le long d'une route sombre traversant le bois de Boulogne, les jeunes femmes déambulent (étrange ballet, filmé à distance par une caméra refusant de trop s'approcher de ses sujets, que celui de ces femmes riantes et moulées dans leurs tenues suggestives, parfois la poitrine découverte) afin d'attirer le regard d'un client potentiel. Il en va de même pour Djamel : c'est bien par le regard que le jeune Beur parvient à " accrocher » certains de ses clients et clientes. La tactique de Djamel s'établit par un contact oculaire, puis par un début de conversation banale, voire cliché ( "vous avez une cigarette?»). Et c'est sans transition aucune que le cinéaste, Sébastien Lifshitz, nous plonge dans la réalité graphique de la vie professionnelle illicite de Djamel : Djamel, dans les toilettes de

${ }^{6}$ Nous empruntons cette expression à Henning Bech (1997, p. 107). 
la gare - les toilettes, espace particulier, car ouvrant « une fuite dans le système clos » (de Certeau, 1980, p. 199) —, avec un homme qui exige de lui une fellation, ou avec une femme qui, visiblement, prend un grand plaisir durant ce moment de promiscuité mais qui semble tout à fait choquée de découvrir que la prestation du jeune homme est tarifée. Au sein de l'espace pratiqué par le groupe dominant, le lieu frontière constitue donc un îlot temporaire pour les membres du groupe dominé, un espace aux fonctions détournées et aux antipodes de l'utilitarisme capitaliste "officiel ». Les toilettes publiques, par exemple, passent de leur fonction de lieu désigné, et seul acceptable, de soulagements corporels spécifiques (l'évacuation d'excréments et d'urine) à celle d'un endroit pouvant accueillir d'autres excrétions et activités corporelles généralement réservées à la sphère privée. Le corps, le regard, la salive, la transpiration, le foutre: le lieu frontière est aussi fondamentalement physique et organique.

\section{Le lieu frontière par opposition à la sphère privée}

Le lieu frontière tel que représenté dans les œuvres du gay noir doit aussi être compris en relation avec la sphère privée, puisque le lieu frontière est en partie constitué par des pratiques généralement dévolues à la sphère privée.

L'Homme blessé, par exemple, établit un contraste marqué entre le lieu frontière homoérotique qu'est la gare nocturne et l'appartement de la famille d'Henri (appartement qui fonctionne comme métonymie de la sphère privée et familiale). Si les deux endroits sont visuellement représentés de manière similaire (des lieux impersonnels aux couleurs ternes, sans 
beauté ou sans grâce), leurs fonctions respectives sont pour Henri bien différentes : s'il existe bien une certaine inquiétude au sein du lieu frontière de la gare (voir ci-dessous), celui-ci est aussi potentiellement le lieu de nouvelles possibilités et de l'espoir d'une connexion avec autrui (dans le cas d'Henri, il s'agit de l'espoir irréaliste de développer une relation avec Jean et de posséder physiquement ce dernier). Mais si Henri se trouve à arpenter de façon quasi obsessionnelle la gare en quête d'un baiser, ou parfois plus, c'est aussi parce qu'il ne peut plus trouver sa place dans le petit appartement de ses parents, ces quelques murs à la décoration médiocre entre lesquels éclatent hystérie familiale et rejet de son prochain. Le père d'Henri est la figure patriarcale par excellence: personnalité monolithique qui n'a aucunement besoin de s'exprimer verbalement pour faire régner l'ordre, son épouse s'en chargeant (par exemple, l'homme est insomniaque et la mère tente de maintenir le silence quand son époux parvient à s'assoupir). De plus, aux dires de la mère d'Henri, les deux adultes, dont la relation semblait battre de l'aile, semblent se retrouver enfin et la femme fournit maints efforts pour conserver cet état de bonheur retrouvé aussi longtemps que possible, quitte à rabrouer périodiquement Henri, de plus en plus instable. L'appartement de la famille d'Henri devient donc rapidement le lieu où se cristallisent les tensions entre une hétéronormativité glaçante ne cessant de s'affirmer et un jeune sujet homosexuel, dans la dissimulation de soi, découvrant à la fois l'aliénation par le biais de la famille et la nécessité de vivre autre chose ailleurs. En ce sens, pour Henri, le lieu frontière est le résultat de la sphère privée hétéronormative.

La sphère privée est aussi représentée dans Wild Side par le biais de l'appartement que partagent Stéphanie, Djamel et 
Mikhail. Contrairement à L'Homme blessé, l'appartement, un lieu modeste désordonné, est ici synonyme d'harmonie polyamoureuse car, à aucun moment, le ménage à trois n'est porteur de jalousie ou de tensions. Au contraire, semble-t-il : le trouple, la configuration sentimentale choisie par Sébastien Lifshitz, et son espace privé évoquent principalement bonheur et satisfaction affective comme sexuelle, tranchant radicalement avec les expériences dépersonnalisées et monnayées de Stéphanie et de Djamel au sein du lieu frontière. Plus problématique est la place des personnages dans la maison de la mère (Josiane Stoléru) de Stéphanie : une demeure délabrée, isolée dans la campagne du Nord, à l'intérieur suranné et désolant reflétant la santé extrêmement fragile de la mère. Loin de Paris, Stéphanie ne peut être elle-même que difficilement dans cette maison remplie des souvenirs de Stéphanie quand celle-ci avait encore un corps de garçon (elle s'appelait alors Pierre), d'une jeune sœur décédée et d'un père dont on ne sait ce qu'il est advenu. Stéphanie, par le biais d'une mère mourante qui voudrait comprendre la vie de sa fille, doit lutter pour ne pas être vue uniquement comme Pierre grimé en femme (d'ailleurs, à la mort de sa mère, Stéphanie jette toutes les photos de famille afin de rompre avec son passé masculin) mais aussi exiger de ses deux conjoints de ne pas coucher dans le même lit sous le toit familial: le regard un peu sévère et incompréhensif de la mère (qui est aussi celui du système patriarcal) pèse.

La sphère privée du trouple, cela dit, existe au-delà du paramètre du petit appartement parisien, et si elle ne trouve pas sa place dans le décor poignant de la maison délabrée de la mère, elle peut se manifester par le biais de tactiques au sein d'espaces publics hétéronormatifs tels que les parcs et les 
transports en commun. Par exemple, Stéphanie, Djamel et Mikhail, étroitement enlacés, roulent les uns sur les autres et descendent un terrain en pente du parc des Buttes-Chaumont. Et c'est bras dessus, bras dessous que le trio s'en va, revendiquant et se réappropriant ainsi, par sa complicité et sa proximité affichées, une partie de cet espace. Il en va de même de la courte scène qui présente Stéphanie, Djamel et Mikhail endormis, serrés les uns contre les autres, dans le compartiment d'un train raccompagnant les trois amoureux à Paris : ici, c'est donc la sphère privée qui voyage dans la sphère publique hétéronormée; c'est la sphère privée (un espace lisse) ${ }^{7}$ qui occupe temporairement et strie l'espace officiel de l'État, ici représenté succinctement par les limites physiques du wagon (qui lui-même, de par son déplacement, strie l'espace campagnard) et du parc des Buttes-Chaumont.

\section{Un lieu d'autosurveillance paranoïaque}

Mais il ne s'agit pas ici d'idéaliser la portée de ces tactiques au sein de l'espace hégémonique de l'hétérosexualité entre autres parce que, comme nous le savons, ces tactiques sont, de par leur nature, temporaires, donc de facto d'une portée restreinte. De Certeau lui-même souligne en effet que les acquis des tactiques ne sont que limités et dépendent d'occasions à saisir dans l'instant (1980, p. 86-7). De plus, les tactiques s'accompagnent aussi de leur corollaire antinomique, à savoir des tentatives de répression potentielles (des contre-stratégies pour ainsi dire)

\footnotetext{
7 Gilles Deleuze et Félix Guattari opposent l'espace lisse à l'espace strié : l'espace lisse est hétérogène et celui du nomade tandis que l'espace strié est homogène et celui du sédentaire et de l’État (1980, p. 434-527).
} 
de la part du groupe dominant. Ici, si le regard continue à jouer un rôle important, il est souvent inquiet, voire paranoïaque.

Tout comme L'Homme blessé et Wild Side, Les Nuits fauves comporte nombre d'exemples où les tactiques des braconniers homosexuels mettent à défi l'espace public hétéronormé : les séquences les plus parlantes sont celles des quais de la Seine ou des lieux de transit nocturnes, où l'on assiste au regroupement nocturne d'hommes dont l'objectif principal est la gratification sexuelle tout aussi ludique qu'immédiate (espace qui, comme le souligne justement Carrie Tarr, permet l'expression et la mise en action de pratiques sexuelles alternatives: 1999, p.117126). Jean circule dans un espace mal éclairé uniquement occupé par des hommes, parfois en couple. Aucun mot n'est prononcé, mais le regard du protagoniste en croise un autre, et il suffit d'un bref instant, le temps de s'appuyer brièvement à un pilier, pour que les deux hommes, auquel se joindra rapidement un troisième individu, s'enlacent; cependant, les sentiments de Jean sont complexes et contradictoires et, comme semble le suggérer le film, la présence du jeune homme dans ces lieux est symptomatique de la confusion émotionnelle du personnage bisexuel, partagé entre son amour pour la jeune Laura (Romane Bohringer) et pour son jeune amant Samy (Carlo Lopez). Jean, en effet, gagne ces lieux frontières sexuels quand son dilemme amoureux (Laura et/ou Samy?) et son trouble existentiel deviennent trop intenses : ici, la relation sexuelle avec d'autres hommes dans le non-lieu nocturne a vocation d'exutoire et d'échappatoire (mais il est tout aussi troublant qu'intéressant de noter que ces mêmes lieux sont occupés - à une occasion, et de jour - par Jean et Laura qui y font l'amour sans gêne aucune ou désir de se cacher). 
Si Les Nuits fauves a marqué son époque par sa représentation frontale du mal-être d'un individu séropositif et de pratiques sexuelles entre hommes dans des lieux publics, cette œuvre n'en reste pas moins problématique dans le lien établi entre les lieux frontières et le sida qui, semble nous dire le cinéaste, est d'abord une maladie accompagnant le monde de la nuit homosexuel et ses pratiques diverses et variées. Les lieux frontières sont donc porteurs de dangers pour soi mais aussi pour autrui : le monde nocturne (la rue, les bars) est aussi peuplé de skin heads racistes (dont Sami qui, de façon incongrue, passe de la découverte du sadomasochisme à l'adhésion à un groupe de l'extrême droite) qui souhaitent en découdre avec un jeune Beur. Mais Jean veille; il se coupe la main puis menace d'infecter le chef des agresseurs avec son sang contaminé, cette arme et cette menace directement issues des lieux frontières nocturnes et identifiées à ceux-ci...

La vigilance est donc nécessaire. Comme il a été suggéré précédemment dans notre description de la séquence de L'Homme blessé durant laquelle Henri et le jeune homme à l'imperméable cherchent un endroit isolé pour s'embrasser, une autosurveillance est également de mise, ou devrait sans doute l'être: Henri ne se fait-il pas berner par Jean lors du cambriolage du magasin de nettoyage à sec quand le malfrat vole la carte d'identité de son partenaire afin de provoquer l'arrestation de celui-ci? L'expression des corps désirants homosexuels dans le lieu frontière est donc parfois empreinte d'une difficulté fondamentale: le regard en recherche un ou plusieurs autres, le corps en recherche et réclame un ou plusieurs autres, mais pas n'importe lequel / lesquels : il faut reconnaître et identifier son prochain en ce qu'il a de similaire à soi (l'homoïté, encore...) tout en restant sur ses gardes afin de 
ne pas côtoyer de trop près son ennemi (qu'il s'agisse de la menace aussi intangible que réelle du sida que celle, physique et violente, des skin heads).

Nous ne saurions dire que, tel que représenté dans les exemples du gay noir composant notre corpus, le lieu frontière s'appuie sur un système de nature panoptique à proprement parler (dans le sens où le regard de surveillance n'est pas centralisé) mais, plutôt, sur un entre-deux : en partie libéré d'un système disciplinaire externe mais aussi dépendant d'une autodiscipline s'opérant par le biais d'un regard de surveillance intériorisé. Le regard du personnage masculin gay (par ailleurs, tout comme les relations masculines individuelles au sein des lieux frontières du gay noir) est en fait de nature rhizomatique ${ }^{8}$, un regard sans cesse en connexion avec d'autres regards, constituant des réseaux de connectivité entre individus (qu'il s'agisse du détenteur d'un regard chargé de menaces comme celui des skin heads ou celui, plus enjôleur, comme celui d'un personnage gay en quête de sa propre reconnaissance et de celle d'autrui).

Mais les menaces qu'accompagnent parfois les lieux frontières peuvent résulter, comme l'illustrent les exemples précédents, en une intériorisation de ce regard consacré à la surveillance (comme le montrent Henri et le jeune homme à l'imperméable dans L'Homme blessé; ou Djamel, en quête prudente d'un client ou d'une cliente dans l'espace principal de la gare du Nord dans Wild Side). Pas besoin d'un édifice

8 « À la différence des arbres ou de leurs racines, le rhizome connecte un point quelconque avec un autre point quelconque [...]. Il n'est pas fait d'unités, mais de dimensions, ou plutôt de directions mouvantes. Il n'a pas de commencement ni de fin, mais toujours un milieu, par lequel il pousse et déborde. » (Deleuze et Guattari, 1980, p. 31) 
tangible, de pierres et de béton, pour exercer une surveillance qui pourrait se rapprocher de celle du panoptique mais un regard tourné vers sa propre personne, dont la fonction perlocutoire serait la surveillance de soi :

Pas besoin d'armes, de violences physiques, de contraintes matérielles. Mais un regard. Un regard qui surveille et que chacun, en le sentant peser sur lui, finira par intérioriser au point de s'observer lui-même. (Foucault, 1994, p. 198)

On ne peut donc surestimer la capacité des braconniers du gay noir pour changer la nature de cet espace ou pour changer les règles du jeu polémologique : si le lieu frontière permet une autre socialité, à sa façon subversive et contestataire, le braconnage du lieu normalement pratiqué par le groupe dominant demeure limité dans le temps et inscrit dans les limites physiques et symboliques du lieu frontière. 


\section{Bibliographie}

AuGÉ, Marc. (1992), Non-Lieux : Introduction à une anthroplogie de la surmodernité, Paris, Seuil.

BECH, Henning. (1997), When Men Meet Men: Homosexuality and Modernity, Cambridge and Chicago, The University of Chicago Press.

BERSANI, Leo. (1996), Homo, Cambridge, Harvard University Press.

Deleuze, Gilles et Félix GuatTARI. (1980), Mille Plateaux: Capitalisme et schizophrénie 2, Paris, Minuit.

Foucault, Michel. (1994), "L'œil du pouvoir», dans Dits et écrits, 1976-1988, Daniel DEFERT et François EWALD (éd.), Paris, Gallimard, p. 190-207.

GrandENA, Florian. (2014), «From the Sublimated Anus to the Desublimating Hand: An Intersectional Discussion of Work and Homosexuality in French Gay Cinema », dans Lucille CAIRNS et Santiago FouZ-HERNANDEZ (dir.), Re-Thinking 'Identities': Western Cultural Articulations of Alterity and Resistance in the New Millennium, Bern, Peter Lang Publishing, p. 89-111.

HoCQUENGHEM, Guy. (1980 [1972]), Le Désir homosexuel, Paris, Fayard.

MARTEL, Frédéric. (1996), Le Rose et le noir, Paris, Seuil.

MiLler, Stephen Paul. (1999), The Seventies Now: Culture as Surveillance, Durham and London, Duke University Press.

PROVENCHER, Denis. (2007), Queer French: Globalization, Language, and Sexual Citizenship in France, Aldershot and Burlington, Ashgate.

-. (2002), "The Next Gene(t)ration», Contemporary French Civilization, vol. 26, no 2, p. 335-346. 
TARR, Carrie. (1999), « Gender and Sexuality in Les Nuits fauves (Collard, 1992)", dans Phil PowRIE (éd.), French Cinema in the 1990s: Continuity and Difference, Oxford, Oxford University Press, p. 117-126.

\begin{abstract}
Résumé
Le cinéma contemporain français à problématique homosexuelle est composé de diverses tendances, dont celle du gay noir, enracinée dans une certaine tradition genétienne qui exige de la figure masculine homosexuelle un penchant certain pour des activités illicites dans des lieux frontières publics nocturnes. En nous inspirant de L'Invention du quotidien de Michel de Certeau (plus précisément de ses concepts de stratégie et de tactique), nous expliquerons que ces lieux frontières sont fondamentalement polémologiques: c'est en effet dans la tension exercée entre stratégies hétéronormatives au sein de l'espace public diurne et tactiques homosexuelles dans un espace public nocturne que le lieu frontière se construit. Ce lieu est aussi de nature physique et organique et construit entre autres par le biais du regard, d'où, parfois, une (auto)surveillance paranoïaque des personnages homosexuels.
\end{abstract}

\begin{abstract}
Contemporary French gay-themed cinema is made up of various trends, such as the Genet-inflected gay noir in which male homosexual figures have a certain penchant for illicit activities within nocturnal public lieux frontières. Drawing from Michel de Certeau's The Invention of Everyday Life (more
\end{abstract}


specifically his concepts of strategy and tactic), I explain that the lieux frontières are fundamentally polemological: indeed, it is the tension between heteronormative strategies in diurnal spaces and homosexual tactics in nocturnal spaces that constructs lieux frontières. I also argue that the lieux frontières are of a physical and organic nature as well as constructed through the gaze (hence an occasional paranoid (self) surveillance enacted by homosexual characters). 